\title{
Role of intermediate states in low-velocity friction between amorphous surfaces
}

\author{
Woo Kyun Kim \\ Department of Aerospace Engineering and Mechanics, University of Minnesota, Minneapolis, Minnesota 55455, USA
}

\author{
Michael L. Falk* \\ Department of Materials Science and Engineering, Department of Mechanical Engineering, and \\ Department of Physics and Astronomy, Johns Hopkins University, Baltimore, Maryland 21218, USA
}

(Received 23 August 2011; published 11 October 2011)

\begin{abstract}
Simulated sliding between an oxidized silicon tip and surface over six decades of velocity using accelerated molecular dynamics (MD) reproduces the experimental velocity dependences of the friction. Unlike in the crystalline case, as increasing forces are applied to the amorphous tip, intermediate states arise. These intermediate states serve as critical transition pathways. The emergence of such states leads to the emergence of a plateau in sliding velocity at lower sliding speeds and higher temperatures. A simple theory based on these observations successfully describes both the experimental and simulated data.
\end{abstract}

DOI: 10.1103/PhysRevB.84.165422

PACS number(s): 68.35.Af, 07.05.Tp, 62.20.Qp, 81.40.Pq

\section{INTRODUCTION}

Friction has presented a challenge since man's earliest technological feats and, in recent years, it has become possible to study nanoscale friction via Atomic Force Microscopy (AFM). ${ }^{1}$ A typical AFM tip is a single asperity contact with a radius of 10 to $100 \mathrm{~nm}^{2}$ Macroscopic friction arises from many such asperities, and as such AFM studies have been used to study superlubricity, ${ }^{3-5}$ the temperature and sliding velocity dependence of friction, ${ }^{6,7}$ and the effects of surface vibrational frequency shifts on nanoscale friction. ${ }^{8}$

Several theoretical models have been developed to interpret the experimental results. ${ }^{9-14}$ However, most of these theoretical models assume idealized crystalline surfaces, ${ }^{9-12}$ although many if not most sliding surfaces are disordered due to native oxides that form spontaneously or are attributable to the extremes of pressure and shear that accompany friction. Recently it has been noticed that transitions in such a system may be significantly more complex than accounted for in the canonical theories of friction. ${ }^{13,15}$ Interesting statistical models for considering the relative roles of bonding and debonding have been proposed, ${ }^{14}$ but such theories would benefit from atomic-scale analysis of particular tip-surface dynamical systems to confirm the mechanisms proposed are, in fact, the most critical.

Directly observing buried sliding interfaces in situ is still a formidable challenge. Molecular dynamics (MD) simulations, ${ }^{16-21}$ including some studies about amorphous contacts, ${ }^{17-19,21}$ have been proposed as an alternative means of observing atomic behaviors, but MD's submicrosecond time scale limits such simulations to sliding speeds orders of magnitude faster than most AFM experiments. One exception is the extension of the parallel replica method to driven systems, ${ }^{22}$ which has reduced sliding velocities as low as $1 \mathrm{~mm} / \mathrm{sec}$. However, this method is generally insufficient to

Published by the American Physical Society under the terms of the Creative Commons Attribution 3.0 License. Further distribution of this work must maintain attribution to the author(s) and the published article's title, journal citation, and DOI. reach experimental sliding speeds in AFM studies, typically in the $\mu \mathrm{m}-\mathrm{nm} / \mathrm{sec}$ range. We demonstrate here that much lower sliding speeds can be achieved using an accelerated MD scheme ${ }^{23}$ based on hyperdynamics. ${ }^{24}$ In this method, which was developed by the authors and applied to a realistic problem for the first time here, the potential energy function is modified in a controlled way to facilitate transitions, and many such simulations are performed simultaneously in order to exploit parallel computing architectures.

Friction between an oxidized silicon AFM tip and surface under ultra-high vacuum conditions has been shown to exhibit unexpected relationships between friction force and sliding velocity with nontrivial temperature dependences. ${ }^{6}$ At lower temperatures the frictional force increases logarithmically with sliding velocity, but at higher temperatures the frictional force had no apparent dependence on the sliding velocity. The simple single-step activated transition picture, the Tomlinson model, ${ }^{9,10}$ has been shown not to hold at low sliding speeds where multistep transition mechanisms must be involved. ${ }^{15}$ However, to the best of our knowledge, there has not been any direct observation of the specific mechanisms that are present during the sliding, although many candidate mechanisms have been proposed. ${ }^{13-15}$ In this study we investigate the origin of these deviations from logarithmic sliding rate dependences predicted by the Tomlinson model using our accelerated MD methodology.

\section{SIMULATION RESULT}

First we present the simulation results and show that these results mirror the experimental observations. ${ }^{6}$ Figure 1 shows our three-dimensional AFM model consisting of an oxidized silicon tip and substrate. The spherical tip with a radius of $2.1 \mathrm{~nm}$ consists of 569 silicon and 309 oxygen atoms, and the substrate $(4.61 \mathrm{~nm} \times 4.61 \mathrm{~nm})$ contains 1152 silicon and 1248 oxygen atoms. The atomic interactions are modeled by a modified Stillinger-Weber potential developed by Watanabe et al. ${ }^{25}$ Instead of including the Coulombic interaction terms, the Watanabe potential models long-ranged interactions with the coordination-dependent $\mathrm{Si}-\mathrm{O}$ pair interaction term and 


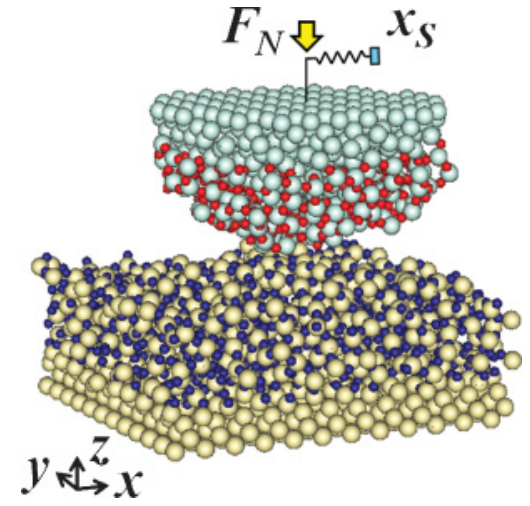

FIG. 1. (Color online) A diagram of a three-dimensional AFM model consisting of an oxidized silicon tip and a substrate. Darkcolored smaller spheres are oxygen atoms, and light-colored larger spheres are silicon atoms.

the three-body term. Moreover, the parameters which appear in these terms were chosen by performing a best fit to the $a b$ initio calculations of various silica clusters, and the Watanabe potential has been used to reproduce the structural features of thin oxide films. ${ }^{25,26}$ The oxidized layers were generated by a procedure proposed by Dalla Torre et al. ${ }^{27}$ in which oxygen atoms are inserted one by one into Si-Si bonds. The tip is joined to the substrate in the [001] direction by a normal force of $1.5 \mathrm{nN}$. The atoms on the bottom of the substrate are fixed, and those on the top of the tip move like a rigid body. These atoms on the top of the tip are pulled by a spring with a stiffness of $6.1 \mathrm{~N} / \mathrm{m}$, representing an AFM cantilever. The normal force and the spring stiffness are derived from experiments in Ref. 6. Temperature is controlled by a Nosé-Hoover chain thermostat, ${ }^{28}$ and the equations of motion are solved using a modified velocity-Verlet algorithm. ${ }^{29}$ To reduce the sliding velocity we used a version of the hyperdynamics method implemented specifically for frictional sliding, as described in our previous work. ${ }^{23}$ Simulations were performed at $100 \mathrm{~K}$ and $400 \mathrm{~K}$ spanning a wide range of sliding velocities from $273 \mathrm{~nm} / \mathrm{sec}$ to $273 \mathrm{~mm} / \mathrm{sec}$.

In Fig. 2(a) the system exhibits a nonuniform stick-slip behavior arising from the amorphous character of the surfaces, which is in contrast to the uniform stick-slip motion observed in MD simulation studies of crystals. ${ }^{22,23}$ In the simulated results, as in the experiments, we clearly observe deviation from the logarithmic velocity dependence of the friction force predicted in the Tomlinson model. Figure 2(b) shows a detailed comparison between the transitions that initiate sliding at $\mathrm{T}=100 \mathrm{~K}$ and $v_{s}=27.3 \mathrm{~mm} / \mathrm{sec}$ and at $\mathrm{T}=400 \mathrm{~K}$ and $v_{s}=$ $2.73 \mu \mathrm{m} / \mathrm{sec}$. Transitions occur among three states: an initial state $\mathrm{A}$, a final state $\mathrm{C}$, and an intermediate state $\mathrm{B}$. The atomic configurations corresponding to these states are illustrated in Fig. 3(a). In state A there is one silicon-silicon bond between the tip and the substrate, and in state B the silicon atom is bonded at a different angle with the same substrate atom. In state $\mathrm{C}$ this silicon atom forms a new bond with a different silicon atom in the substrate. The transition from $B$ to $C$ induces a larger drop in the lateral force than the transition from A to B. While at low temperature the system undergoes two one-way transitions (first from A to B and then from B to C); at high temperature the system switches back and forth between $\mathrm{A}$ and $\mathrm{B}$, confirming the importance of multistep processes, as noted in previous investigations by others. ${ }^{13,15}$ In fact the frequency of the transitions was greater than apparent in Fig. 2(b) since limited resolution requires us to show time-averaged data.

Figures 3(b) and 3(c) show the tip positions and the minimum energies of each state as a function of the slider position and illustrate that the stability of each state depends on the slider position. The intermediate state $\mathrm{B}$, in particular, does not emerge until a finite force is applied. This is a significant additional deviation from the Tomlinson model not anticipated
$\mathrm{V}_{\mathrm{s}}=27.3 \mathrm{~mm} / \mathrm{sec}$ and $\mathrm{T}=100 \mathrm{~K}$

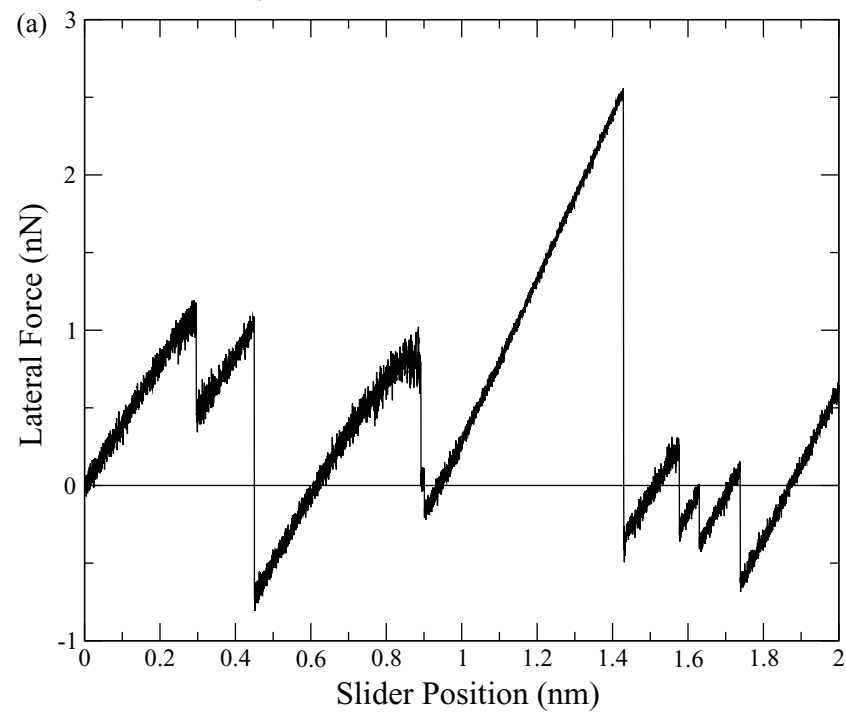

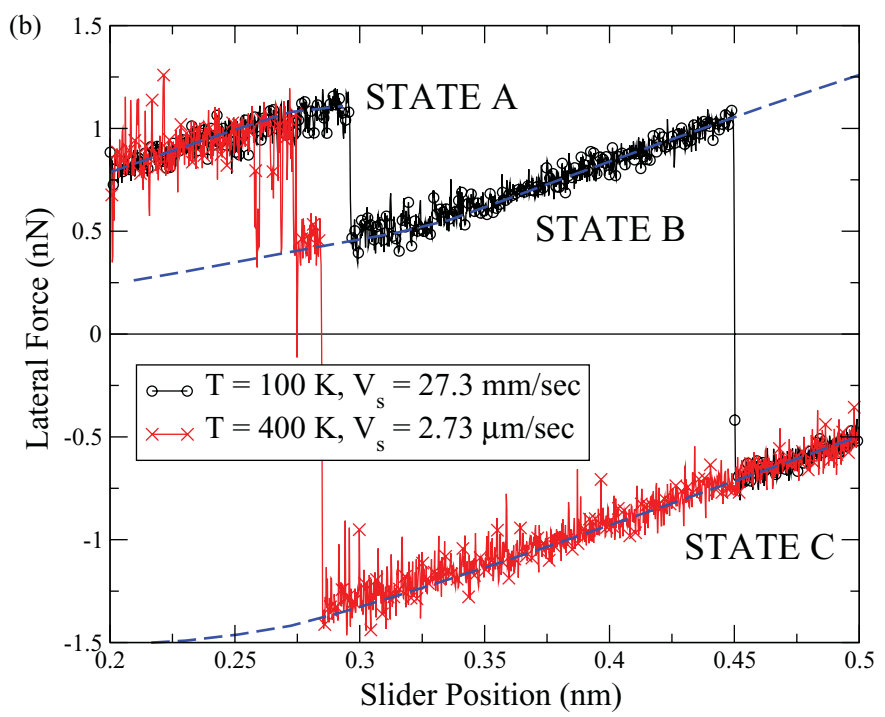

FIG. 2. (Color online) (a) The lateral force as a function of slider position measured at $v_{s}=27.3 \mathrm{~mm} / \mathrm{sec}$ and $\mathrm{T}=100 \mathrm{~K}$. (b) Lateral forces near the first transition as functions of the slider position. Dashed lines represent the three states illustrated in Fig. 3. 
(a)

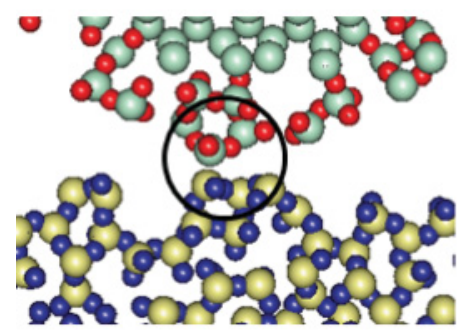

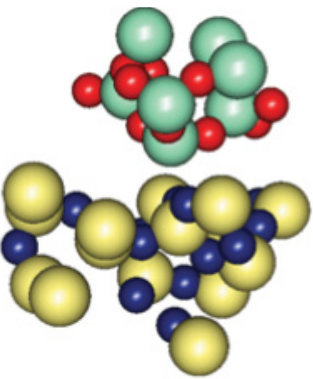

STATE A

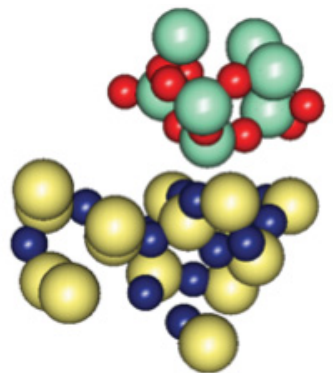

STATE B

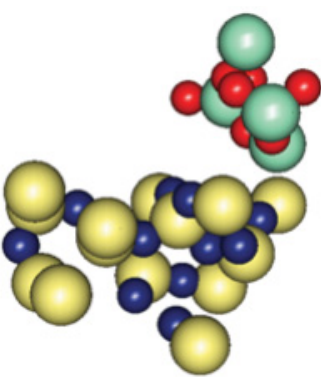

STATE C (b)

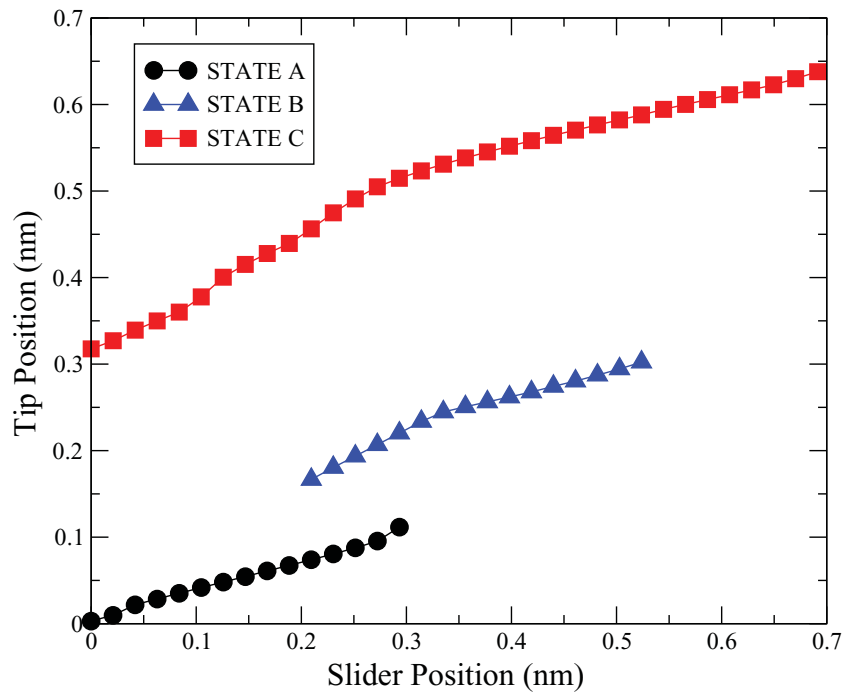

(c)

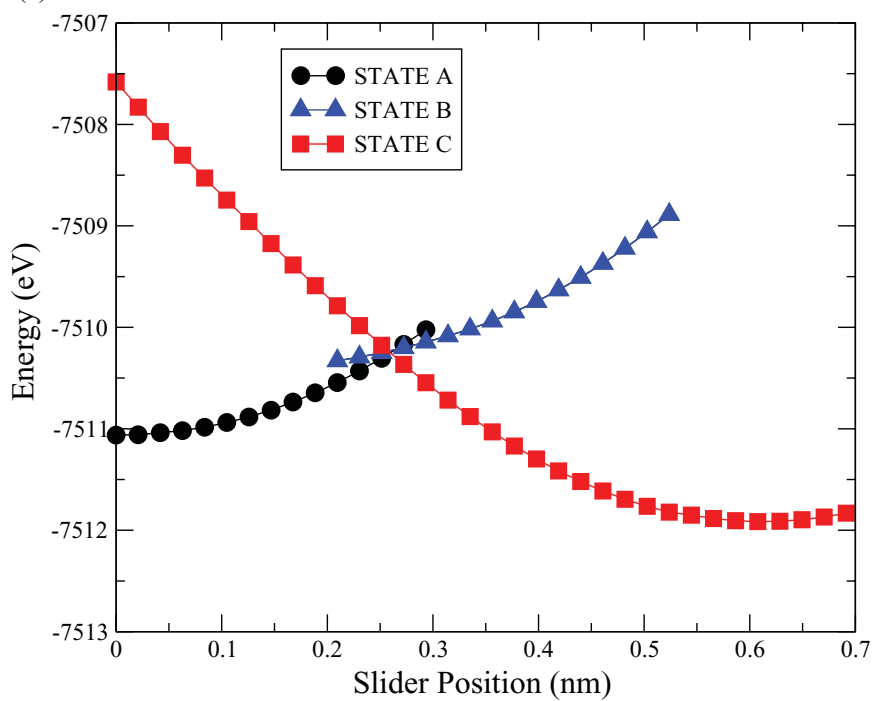

FIG. 3. (Color online) (a) Atomic configurations of states A, B, and C. (b) The tip positions of the minimized configurations corresponding to each state as functions of the slider position. (c) The minimum energies of each state as functions of the slider position.

in prior work, such as Refs. 13 and 15. At the smaller slider positions only states $\mathrm{A}$ and $\mathrm{C}$ are stable. As the slider moves, state B emerges, and it becomes more stable than state A after a slider displacement of $0.26 \mathrm{~nm}$. As the slider moves further, state $\mathrm{A}$ becomes unstable, and states $\mathrm{B}$ and $\mathrm{C}$ remain. Neither the transition from $\mathrm{A}$ to $\mathrm{C}$ nor the backward transition from $\mathrm{C}$ to $\mathrm{B}$ is observed.

\section{THEORETICAL MODEL}

The previous observations, taken together, imply that switching between states A and B has a significant effect on the lateral force in large part because of the intermediate nature of state B and its absence at low applied loads. If the transition occurs when only states B and C exist, as would happen at high sliding speeds and/or low temperatures, we should see the predicted logarithmic dependence. ${ }^{10}$ At sufficiently low sliding speed and/or high temperature, A and B coexist. As the sliding speed decreases and/or the temperature rises, the probability of residing in the intermediate state $\mathrm{B}$ decreases, and the rate of a transition to state $\mathrm{C}$ consequently decreases leading to a deviation from the logarithmic dependence. Since state B does not exist until the system achieves a certain slider position, no matter how slow the sliding speed or high the temperature a critical force must be reached before the transition can proceed along this pathway.
To analyze this phenomenon quantitatively we have developed a theoretical model. We begin by writing a set of Kramers' rate equations ${ }^{30}$ among states $\mathrm{A}, \mathrm{B}$, and $\mathrm{C}$. Using the linear relationship between the lateral force and the slider position ( $\left.F=k_{\text {eff }} x_{s}=k_{\text {eff }} v_{s} t\right)$, we obtain the rate equations in terms of the lateral force, as in Gnecco's model ${ }^{10}$

$$
\begin{gathered}
\frac{d p_{A}}{d F}=-\frac{R_{A \rightarrow B}(F)}{k_{\mathrm{eff}} v_{S}} p_{A}+\frac{R_{B \rightarrow A}(F)}{k_{\mathrm{eff}} v_{S}} p_{B} \\
\frac{d p_{B}}{d F}=+\frac{R_{A \rightarrow B}(F)}{k_{\mathrm{eff}} v_{S}} p_{A}-\left(\frac{R_{B \rightarrow A}(F)+R_{B \rightarrow C}(F)}{k_{\mathrm{eff}} v_{S}}\right) p_{B},
\end{gathered}
$$

and

$$
\frac{d p_{C}}{d F}=+\frac{R_{B \rightarrow C}(F)}{k_{\mathrm{eff}} v_{S}} p_{B},
$$

where $p_{A}, p_{B}$, and $p_{C}$ are the probabilities that the system stays at $\mathrm{A}, \mathrm{B}$, and $\mathrm{C}$, respectively, and $R_{A \rightarrow B}, R_{B \rightarrow A}$, and $R_{B \rightarrow C}$ are the transition rates. We ignore the backward transitions from $\mathrm{C}$ to $\mathrm{A}$ or $\mathrm{B}$ and the transition from $\mathrm{A}$ to $\mathrm{C}$. We write the rate constants in the standard Arrhenius form, $f \exp (-\beta \Delta \mathrm{E})$ where $\beta=1 / k_{B} \mathrm{~T}$. We make the approximation that $R_{A \rightarrow B}$, $R_{B \rightarrow A} \gg R_{B \rightarrow \mathrm{C}}$ so that the system, on average, makes many more transitions between $\mathrm{A}$ and $\mathrm{B}$ before making the transition 

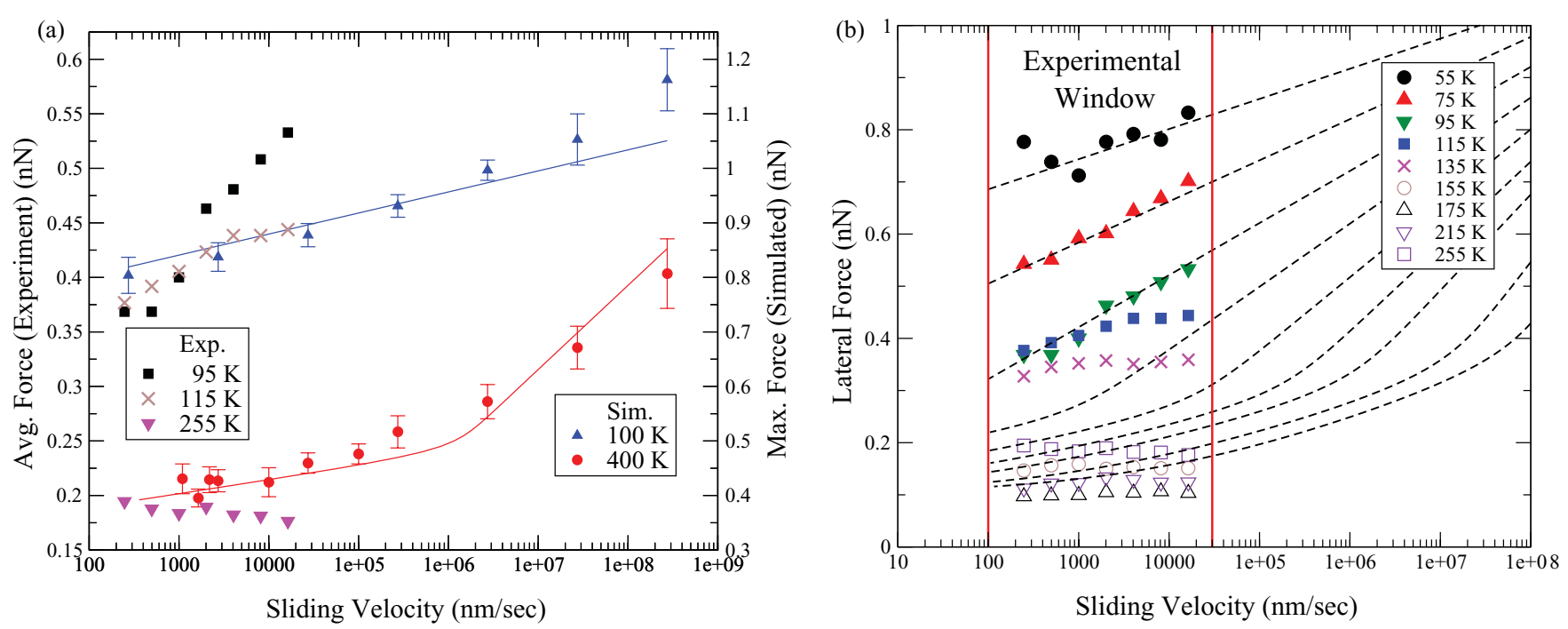

FIG. 4. (Color online) Lateral forces as functions of sliding velocity. (a) Simulation ( $100 \mathrm{~K}$ and $400 \mathrm{~K}$ ) in comparison with the experimental data from Ref. $6(95 \mathrm{~K}, 115 \mathrm{~K}$, and $255 \mathrm{~K})$. The error bars represent the standard deviations. (b) AFM experiment (Ref. 6) over a wide range of temperatures and at sliding velocities shown in the experimental window. Solid curves in (a) and dashed curves in (b) are fits based on the intermediate state switching theory discussed in the text.

from $\mathrm{B}$ to $\mathrm{C}$, leading to the relation

$$
\frac{p_{B}}{p_{A}+p_{B}} \approx \frac{R_{A \rightarrow B}}{R_{A \rightarrow B}+R_{B \rightarrow A}}=\frac{1}{1+\eta(F) \exp \left[\beta\left(V_{B}-V_{A}\right)\right]},
$$

where $V_{A}$ and $V_{B}$ are the energies of state $\mathrm{A}$ and $\mathrm{B}$. The function $\eta(F)$ is the ratio of the attempt frequencies of the $B \rightarrow A$ and $A \rightarrow B$ transitions. We approximate this ratio as

$$
\eta(F)=C_{1}\left[\left(F-F_{U}\right) /\left(F-F_{L}\right)\right]^{2},
$$

where the $A \rightarrow B$ and $B \rightarrow A$ attempt frequencies must vanish at some lower critical force $F_{L}$ and upper critical force $F_{U}$, respectively, since only state $\mathrm{A}$ is stable when $F<F_{L}$, only state $\mathrm{B}$ is stable when $F>F_{U}$, and both are stable when $F_{L}<F<F_{U}$. To obtain an explicit expression for the most probable force at transition $F^{*}$, we assume that the transition energies vary linearly with the applied force such that $\Delta \mathrm{E}_{B \rightarrow C}$ $=\lambda\left(F_{C}-F\right)$, where $F_{C}$ is the lateral force when the energy barrier from $B$ to $C$ vanishes. We also parameterize the energy difference between states $\mathrm{A}$ and $\mathrm{B}$ in terms of the applied force by the linear expression $V_{B}-V_{A}=C_{2} F+C_{3}$. From Eqs. (3), (4), and (5) the probability distribution function of the lateral force at transition, $g(F)$ is given by

$$
g(F)=-\frac{d\left(p_{A}+p_{B}\right)}{d F}=\frac{d p_{C}}{d F}=\frac{R_{\mathrm{eq}}}{k_{\mathrm{eff}} v_{S}} \times\left(p_{A}+p_{B}\right),
$$

where

$$
R_{\text {eq }}=\frac{R_{B \rightarrow C} \times R_{A \rightarrow B}}{R_{A \rightarrow B}+R_{B \rightarrow A}}=\frac{f_{B \rightarrow C} \exp \left[-\beta \Delta E_{B \rightarrow C}\right]}{1+\eta \exp \left[\beta\left(V_{B}-V_{A}\right)\right]} .
$$

Then the most probable lateral force at transition $F^{*}$, satisfying $g^{\prime}\left(F^{*}\right)=0$ is obtained by solving the following equation:

$$
\left.\frac{d R_{\mathrm{eq}}}{d F}\right|_{F^{*}}=\frac{\left(R_{\mathrm{eq}}\right)^{2}}{k_{\mathrm{eff}} v_{S}} .
$$

By imposing this condition and incorporating the relationships $\Delta \mathrm{E}_{B \rightarrow C}=\lambda\left(F_{C}-F\right)$ and $V_{B}-V_{A}=C_{2} F+C_{3}$, we can derive the sliding speed as a function of the temperature and friction force,

$$
\frac{v_{S}}{v_{O}}=\frac{\exp \left[\lambda \beta\left(F^{*}-F_{C}\right)\right]}{1+\left[\left(1-\frac{C_{2}}{\lambda}\right) \eta-\frac{\eta^{\prime}}{\lambda \beta}\right] \exp \left[\beta\left(C_{2} F^{*}+C_{3}\right)\right]},
$$

where

$$
v_{O}=\frac{f_{B \rightarrow C}}{\lambda \beta k_{\mathrm{eff}}} .
$$

Note that when only states $\mathrm{B}$ and $\mathrm{C}$ exist, we recover a logarithmic dependence, as in Gnecco's model, ${ }^{10}$ and Eq. (9) simply becomes $F^{*}=(1 / \lambda \beta) \ln \left(v_{s} / v_{o}\right)+F_{C}$.

Now we analyze both the simulation results and the experimental observations using Eq. (9). The comparison of the theory and the simulation is shown in Fig. 4(a) using fit parameters from Table I. Most parameters are determined directly from Fig. 3 and the high-velocity portion of the data leaving only one independent fitting parameter. It is apparent that the switching between states $\mathrm{A}$ and $\mathrm{B}$, modeled in the denominator of Eq. (9), results in a plateau at the higher temperature. We expect this theory is too simple to accurately model the experiments that represent an average over many dissimilar transitions. However, if such state switching is common, and if the energies associated with such transitions are not broadly distributed, the model should be able to capture the general trends in the data. Figure 4(a) also shows the force at the initiation of sliding in comparison with the experimental data from Ref. 6. The forces measured in simulation are the peak force during a single slip transition, while the experiments measure average sliding force over several nanometers. For this reason we expect the two quantities to be related by a factor of 2 . 
TABLE I. Fitting parameters.

\begin{tabular}{lcc}
\hline \hline Parameters & Simulation & Experiment \\
\hline$\lambda(\AA)$ & 0.828 & 0.302 \\
$F_{C}(\mathrm{nN})$ & 1.09 & 1.15 \\
$F_{L}(\mathrm{nN})$ & 0.25 & 0.1 \\
$F_{U}(\mathrm{nN})$ & 0.6 & 0.6 \\
$F_{C} \mathrm{f}_{B \rightarrow C} / k_{\text {eff }}(\mathrm{m} / \mathrm{sec})$ & 142 & 572 \\
$C_{1}$ & 120 & 10 \\
$C_{2}(\mathrm{eV} \mathrm{nN})$ & -1.80 & -0.25 \\
$C_{3}(\mathrm{eV})$ & 0.681 & 0.01 \\
\hline \hline
\end{tabular}

Figure 4(b) shows an application of the theory to describe the lateral forces measured by AFM in Ref. 6 over a wide range of temperatures $(55 \sim 255 \mathrm{~K})$ and at the sliding velocities ranging from $100 \mathrm{~nm} / \mathrm{sec}$ to $16 \mu \mathrm{m} / \mathrm{sec}$. The fit parameters used in Fig. 4(b) are given in Table I. The general trend of decreasing force and decreasing velocity dependence with increasing temperature is captured by the theory. More importantly, the transition from logarithmic dependence at low temperature to a high temperature plateau is consistent with predictions based on the theory. The nonmonotonic dependence of force on temperature at high temperature, we believe, may arise from experimental limits accurately resolving such small differences in force. The model also predicts that at higher sliding velocity the logarithmic dependence will be recovered at higher temperatures.

*mfalk@jhu.edu

${ }^{1}$ G. Binnig, C. F. Quate, and Ch. Gerber, Phys. Rev. Lett. 56, 930 (1986).

${ }^{2}$ R. W. Carpick and M. Salmeron, Chem. Rev. 97, 1163 (1997).

${ }^{3}$ M. Hirano, K. Shinjo, R. Kaneko, and Y. Murata, Phys. Rev. Lett. 67, 2642 (1991).

${ }^{4}$ M. Dienwiebel, G. S. Verhoeven, N. Pradeep, J. W. M. Frenken, J. A. Heimberg, and H. W. Zandbergen, Phys. Rev. Lett. 92, 126101 (2004).

${ }^{5}$ A. Socoliuc, R. Bennewitz, E. Gnecco, and E. Meyer, Phys. Rev. Lett. 92, 134301 (2004).

${ }^{6}$ A. Schirmeisen, L. Jansen, H. Hölscher, and H. Fuchs, Appl. Phys. Lett. 88, 123108 (2006).

${ }^{7}$ X. Zhao, M. Hamilton, W. G. Sawyer, and S. S. Perry, Tribol. Lett. 27, 113 (2007).

${ }^{8}$ R. J. Cannara, M. J. Brukman, K. Cimatu, A. V. Sumant, S. Baldelli, and R. W. Carpick, Science 318, 780 (2007).

${ }^{9}$ G. A. Tomlinson, Philos. Mag. 7, 905 (1929).

${ }^{10}$ E. Gnecco, R. Bennewitz, T. Gyalog, Ch. Loppacher, M. Bammerlin, E. Meyer, and H.-J. Güntherodt, Phys. Rev. Lett. 84, 1172 (2000).

${ }^{11}$ Y. Sang, M. Dube, and M. Grant, Phys. Rev. Lett. 87, 174301 (2001).

${ }^{12}$ S. Yu. Krylov, K. B. Jinesh, H. Valk, M. Dienwiebel, and J. W. M. Frenken, Phys. Rev. E 71, 065101 (2005).

${ }^{13}$ D. G. Abel, S. Yu. Krylov, and J. W. M. Frenken, Phys. Rev. Lett. 99, 166102 (2007).

${ }^{14}$ I. Barel, M. Urbakh, L. Jansen, and A. Schirmeisen, Phys. Rev. Lett. 104, 066104 (2010).

\section{CONCLUSION}

In conclusion we showed using a novel-accelerated MD scheme that switching between states, combined with the emergence of new intermediate states during sliding, together plays a crucial role in determining the forces between amorphous surfaces. This work demonstrates the impact that simulation methodologies capable of reaching longer time scales can have in the interpretation of nanoscale experimental investigations. Moreover, it is striking that even a rather simple theoretical model that includes this mechanism is able to explain the observations in an actual experimental system analytically over a wide range of sliding speeds and temperatures. Although the sliding model used in this study is not completely realistic in terms of size, contact area, scanning length, etc., we conjecture that this type of state-switching mechanism may be quite common in frictional sliding especially when one or more of the involved surfaces are not perfectly crystalline. If this is indeed true, then our model is more applicable than the Tomlinson-derived models that assume a unidirectional transition mechanism.

\section{ACKNOWLEDGMENTS}

We acknowledge support of the NSF program on Materials and Surface Engineering under Grant Nos. CMMI-0510163 and CMMI-0926111 and the use of facilities at the Johns Hopkins University Homewood High Performance Compute Cluster. We also thank A. Schirmeisen and M.O. Robbins for useful discussions.
${ }^{15}$ M. Evstigneev, A. Schirmeisen, L. Jansen, H. Fuchs, and P. Reimann, Phys. Rev. Lett. 97, 240601 (2006).

${ }^{16}$ G. He and M. O. Robbins, Tribol. Lett. 10, 7 (2001).

${ }^{17}$ L. Wenning and M. H. Müser, Europhys. Lett. 54, 693 (2001).

${ }^{18}$ G. T. Gao, P. T. Mikulski, and J. A. Harrison, J. Am. Chem. Soc. 124, 7202 (2002).

${ }^{19}$ B. Luan and M. O. Robbins, Nature 435, 929 (2005).

${ }^{20}$ J.-H. Wu, S. Karthikeyana, M. L. Falk, and D. A. Rigney, Wear 259, 744 (2005).

${ }^{21}$ M. Chandross, Ch. D. Lorenz, M. J. Stevens, and G. S. Grest, Langmuir 24, 1240 (2008).

${ }^{22}$ A. Martini, Y. Dong, D. Perez, and A. F. Voter, Tribol. Lett. 36, 63 (2009).

${ }^{23}$ W. K. Kim and M. L. Falk, Model. Simul. Mater. Sc. 18, 034003 (2010).

${ }^{24}$ A. F. Voter, J. Chem. Phys. 106, 4665 (1997).

${ }^{25}$ T. Watanabe, H. Fujiwara, H. Noguchi, T. Hoshino, and I. Ohdomari, Jpn. J. Appl. Phys. 38, L366 (1999).

${ }^{26} \mathrm{~T}$. Watanabe and I. Ohdomari, Thin Solid Films 343, 370 (1999).

${ }^{27}$ J. Dalla Torre, J.-L. Bocquet, Y. Limoge, J.-P. Crocombette, E. Adam, G. Martin, T. Baron, P. Rivallin, and P. Mur, J. Appl. Phys. 92, 1084 (2002).

${ }^{28}$ G. J. Martyna, M. L. Klein, and M. Tuckerman, J. Chem. Phys. 97, 2635 (1992).

${ }^{29}$ G. J. Martyna, M. E. Tuckerman, D. J. Tobias, and M. L. Klein, Mol. Phys. 87, 1117 (1996).

${ }^{30}$ H. A. Kramers, Physica 7, 284 (1940). 